\title{
The Control of Foot Placement During Compensatory Stepping Reactions: Does Speed of Response Take Precedence over Stability?
}

\author{
Brian E. Maki, Member, IEEE, and William E. McIlroy
}

\begin{abstract}
Rapid, reflex-like stepping movements are a prevalent and functional compensatory reaction to destabilization, however, little is known about the underlying control. In this paper, a model is developed to examine how speed and stability demands affect control of foot placement during forward and backward compensatory stepping reactions. The concept of the velocity stability margin (VSM) is introduced to characterize the degree to which the horizontal velocity of the falling body approaches biomechanical limits on the capacity to decelerate the center of mass; analogous limits on center-of-mass displacement are quantified in terms of the displacement stability margin (DSM). The model is used to predict, for any initial step characteristics, the variation in DSM and VSM that would occur as a function of changes in timing of foot placement. The VSM was found to prevail over the DSM in establishing limits of stability. Model simulations demonstrated that there typically exists a minimum swing duration that maximizes speed of response while meeting minimum requirements for stability (VSM $\geq 0$ ), as well as a slower speed of response (longer swing duration) at which stability (VSM) is maximized. Experimental data from platform-perturbation tests in 20 healthy young (22-28) and older (65-81) adults were used, in conjunction with the model, to investigate whether speed or stability takes precedence during natural behavior. Control of single-step reactions appeared to favor stability; although the model predicted that a minimally stable step (VSM $=0$ ) could be attained by swing durations as short as $30 \mathrm{~ms}$, the observed swing durations were, on average, $135 \mathrm{~ms}$ longer than this, and the average VSM was nearly as large $(80 \%)$ as the optimally stable value predicted by the model. Control of the initial step of multiple-step reactions was distinctly different. The average swing duration was only $55 \mathrm{~ms}$ greater than the minimally stable value and the average VSM was $81 \%$ smaller than in the single-step reactions. This reduction in VSM is consistent with a need to execute additional steps and appears to support the validity of the model. This model may help to provide insight into the biomechanical factors that govern the neural control of compensatory stepping reactions.
\end{abstract}

Index Terms-Balance, optimization, postural control, stability, stepping.

Manuscript received June 23, 1998; revised December 8, 1998. This work was supported by an operating grant from the Medical Research Council of Canada (\#MT-13355).

B. E. Maki is with the Centre for Studies in Aging, Sunnybrook and Women's College Health Sciences Centre and the Department of Surgery and Institute of Medical Science, University of Toronto, Toronto, Ont. M4N 3M5 Canada.

W. E. McIlroy is with the Centre for Studies in Aging, Sunnybrook and Women's College Health Sciences Centre and the Graduate Department of Rehabilitation Science, University of Toronto, Toronto, Ont. M4N 3M5 Canada.

Publisher Item Identifier S 1063-6528(99)02228-4.

\section{NOMENCLATURE}

$\begin{array}{ll}\text { a-p } & \text { Antero-posterior. } \\ \text { BOS } & \text { Base of support. } \\ \text { COP } & \text { Center of foot pressure. } \\ \text { COM } & \text { Center of body mass. } \\ \text { CNS } & \text { Central nervous system. } \\ \text { DSM } & \text { Displacement stability margin. } \\ \text { FO } & \text { Foot off. } \\ \text { FC } & \text { Foot contact. } \\ \text { VSM } & \text { Velocity stability margin. } \\ a & \text { Antero-posterior platform acceleration. } \\ \text { COP } & \text { Maximum antero-posterior center-of-pressure } \\ & \text { displacement. } \\ D_{\text {a-p COM }} & \text { Antero-posterior center-of-mass displacement. } \\ F_{y} & \text { Antero-posterior ground reaction force. } \\ F_{z} & \text { Vertical ground reaction force } \\ g & \text { Acceleration due to gravity. } \\ h & \text { Height of ankle axis above support surface. } \\ J & \text { Rotational inertia of body with respect to ankle } \\ & \text { axis. } \\ L & \text { Distance between center of mass and ankle axis. } \\ m & \text { Body mass. } \\ \Theta & \text { Body angle (i.e., angle of inverted pendulum). } \\ & \text { Flexion-extension ankle moment ("torque"). }\end{array}$

\section{INTRODUCTION}

$\mathbf{R}$ ECENT studies have shown that rapid, reflex-like stepping movements are a very prevalent compensatory reaction to destabilization during upright stance, even when the postural disturbance is relatively small [1]-[3]. While the much-studied "automatic postural responses" [4] that act to counter undesired displacement of the center of mass (COM) may provide an early defense against instability, change in the base of support (BOS), via compensatory stepping movements, ultimately has the potential to make a much larger contribution to stabilization [3]. In view of recent evidence of age-related impairments in the control of compensatory stepping [5]-[9], it appears likely that the ability to generate these stepping reactions, rapidly and effectively, may be of paramount importance in preventing loss of stability and falls.

Although there is an increasing awareness of the importance of studying compensatory stepping, there is, at present, very limited understanding of the control of these reactions. Critical 
control parameters include 1) swing-leg selection, 2) timing of key events (response initiation, swing-leg unloading, footoff and foot-contact), and 3) spatial characteristics of the foot trajectory. This array of control variables appears to provide considerable redundancy, i.e., for a given perturbation to the COM, there exist numerous combinations of step parameters that will reestablish postural equilibrium. Given these redundant "degrees of freedom," it is not at all clear how the central nervous system (CNS) acts to select a specific response.

The control of the stepping reaction is further complicated when the characteristics of the postural perturbation are unpredictable. This would appear to preclude the possibility of preplanning an effective stepping reaction, forcing the CNS instead to control the step on the basis of ongoing sensory inflow. If the step is to be initiated rapidly, it may be necessary to select many of the step parameters (e.g., swing leg, initial step direction, and velocity) on the basis of incomplete information about the state of instability, and the CNS must somehow deal with the fact that the perturbation may continue to affect the motion of the COM, in an unpredictable manner, after the step has been initiated or even after the step has been completed. Inaccuracies in the internal CNS representation of the COM position and motion, relative to the BOS, may exacerbate the control problem.

Given such uncertainty, a possible control strategy is to use the available information to try to maximize the "stability margins" associated with the initial step. Such a strategy would increase the likelihood that the step will provide sufficient stabilization to recover equilibrium despite ongoing, unpredictable perturbation and an absence of complete or accurate information about the postural state. However, speed of response may also be important, particularly if the initial step fails to recover equilibrium. A faster initial step will increase the time available to execute further stepping reactions before the COM has been displaced to such an extent that recovery of balance is no longer possible. By facilitating execution of multiple steps, rapid stepping may allow "more adjustments to be made to correct for ill-chosen early responses" [7].

In this paper, a biomechanical model is developed to examine how speed and stability demands could affect the control of foot placement during compensatory stepping. In contrast to previous models of foot-placement control that have focussed on volitional step initiation [10], steady-state gait [11]-[14] or obstacle avoidance [15], [16], the present model deals with the control of foot placement during the rapid reflex-like reactions that serve to recover postural equilibrium. To simplify the model, we limit consideration to forward and backward stepping and neglect demands related to lateral stability. Three main research questions are addressed. First, the model is used to explore the tradeoffs between speed and stability, and to determine whether maximizing the speed of the stepping response is compatible with maximizing the stability of the step. We then use experimental data, in conjunction with the model, to determine whether speed or stability takes precedence during natural postural behavior. Finally, these experimental data are used to determine whether decrease in the stability of the initial step is, as predicted by the model,

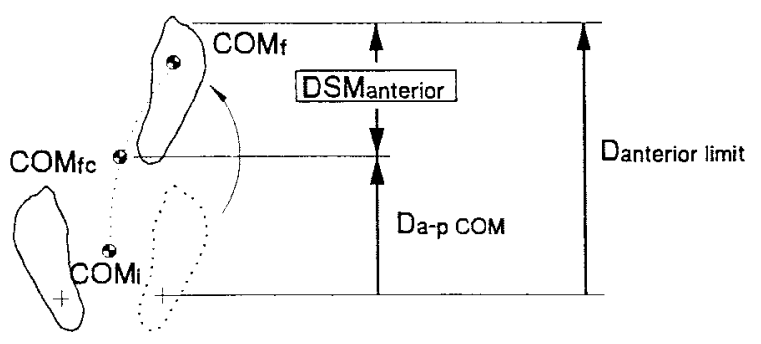

(a)

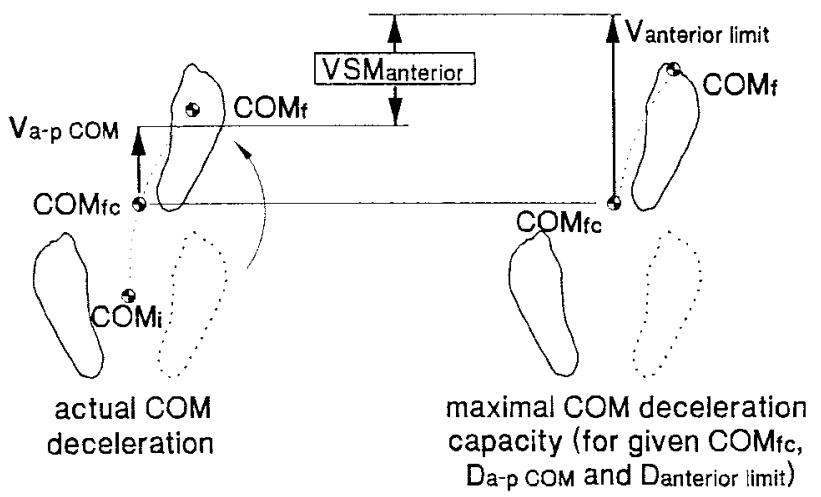

(b)

Fig. 1. Definition of the stability margins and associated terminology [see (1) and (2)]: (a) displacement stability margin (DSM) and (b) velocity stability margin (VSM). The drawings depict the anterior stability margins associated with a forward step; analogous definitions apply to backward steps. $\mathrm{COM}_{i}, \mathrm{COMf}_{c}$, and $\mathrm{COM}_{f}$ represent the position of the center of mass initially (prior to perturbation), at time of foot-contact and at final equilibrium $(\mathrm{COM}$ velocity $=0)$, respectively. $V_{\mathrm{a}-\mathrm{p}} \mathrm{COM}$ is the actual antero-posterior velocity of the COM at time of foot-contact, whereas $V_{\text {anterior limit }}$ is the maximum antero-posterior COM velocity that could have been decelerated (COM velocity $\rightarrow 0$ as the COM reaches end of base of support).

predictive of the need to take further stabilizing action, i.e., additional steps.

\section{Biomechanical Requirements For a Stable SteP}

One model that has been proposed for the control of stepping during gait involves a symmetrical positioning of the swing foot and stance foot, with respect to the pelvis, at time of foot-contact [13]. Such a control scheme serves to provide stability during weight transfer by centering the COM over the BOS. It seems unlikely, however, that this control strategy would apply to compensatory stepping, which involves task objectives that are quite different in some respects. In particular, stepping during gait must preserve the forward momentum of the body, whereas stepping to recover balance is aimed at halting the falling motion of the body.

A necessary, but not sufficient, requirement for arresting a fall by means of a single stepping movement is that the new BOS stability boundary established by the contacting foot "captures" the COM, i.e., the displacement stability margin (DSM) must be greater than or equal to zero. For example, to prevent a forward fall

$$
\mathrm{DSM}_{\text {anterior }}=D_{\text {anterior limit }}-D_{\text {ap COM }}>0
$$

where the variables refer to the values at time of foot-contact [see Fig. 1(a)]. A further requirement is that the stabilizing 
horizontal impulse (i.e., the time-integral of the net horizontal ground reaction force) is sufficient to arrest the horizontal momentum of the falling body before the COM reaches the BOS stability boundary. Analogous to the DSM, this requirement can be expressed in terms of a velocity stability margin (VSM), which quantifies the difference between the velocity stability boundary (i.e., the maximum COM velocity that could be decelerated given the relative positions of the COM and BOS at time of foot-contact) and the actual COM velocity occurring at time of foot-contact. For example, in the case of forward falls

$$
\mathrm{VSM}_{\text {anterior }}=V_{\text {anterior limit }}-V_{\mathrm{a}-\mathrm{p} \mathrm{COM}}>0 .
$$

Again, the variables refer to the values at time of foot-contact [see Fig. 1(b)].

Control of a step so as to decelerate the falling body involves a complex interaction of effects. As noted above, the ability to decelerate a given COM velocity is dependent on the capacity to generate shear impulse, which in turn depends on the magnitude of the shear force $\left(F_{\mathrm{a}^{-} \mathrm{p}}\right)$ that can be generated and the time interval $(\Delta t)$ available for this force to act, before the COM reaches the BOS stability limits. For example, for forward falls

$$
V_{\text {anterior limit }}=(1 / m) \int_{t o}^{t o+\Delta t} F_{\mathrm{a}^{-} \mathrm{p}}(t) d t
$$

where $t_{O}=$ time of foot-contact and $\mathrm{m}$ is the mass of the body. Changes in step parameters that act to increase the DSM, at time of foot-contact, will also act to increase the VSM by increasing the maximum velocity that can be

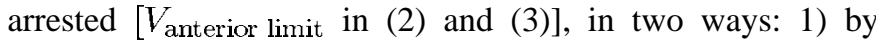
allowing larger shear force to be generated at the contacting foot (an inverted-pendulum model shows that the shear force is approximately proportional to the horizontal distance between the COM and the center of foot pressure (COP) [17]) and 2) by increasing the time available for the shear force to act. Conversely, these same changes in step parameters can adversely affect stabilization by increasing the COM velocity that has to be arrested $\left[V_{\mathrm{a}-\mathrm{p} C O M}\right]$ in (2). For example, a prolonged swing duration can lead to increased step length and concomitant increase in DSM; however, the delay in achieving foot-contact also allows more time for the falling motion of the body to accelerate. The potential tradeoff of effects implies that there may exist a set of optimal step parameters that will maximize the VSM, for any given set of perturbation characteristics and "initial" COM conditions (i.e., COM position and velocity at time of foot-off).

\section{THE BIOMECHANICAL MODEL}

The data from an experimental trial can only be used to determine the DSM and VSM associated with the particular combination of step parameters that were used in that trial. A biomechanical model was developed in order to examine how the DSM and VSM would change as a function of changes in the timing of the foot placement. Specifically, the biomechanical model predicts the antero-posterior (a-p) trajectory of the COM and the concomitant displacement of the swing foot, for a range of potential foot-contact (FC) times. In essence, the model is used to extrapolate beyond the experimentally measured FC time.

\section{A. Swing Phase Simulation}

It was assumed that a resisted "free-fall" follows footoff (FO), i.e., the body falls forward or backward under the influence of gravity, with resistance provided primarily by the stance-foot ankle musculature. For each trial, the experimentally measured data were used to determine the horizontal COM and foot trajectories up to the time of actual FC, and the model simulation was used to extrapolate these trajectories beyond the measured FC time. Measured values for COM position and velocity at time of actual FC, average stance-leg ankle torque during swing phase and average swingfoot velocity provided the "initial" conditions and control parameters needed for the swing-phase simulation. By examining the effect of increasing or decreasing the FC time (or, equivalently, swing duration), while holding all other control parameters constant, the simulations allowed us to explore the extent to which the actual FC times were consistent with maximization of speed of response and/or stability.

In order to predict the swing-phase COM trajectory, the dynamics of the body were approximated by a single-link inverted pendulum, with stabilizing moment generated at the ankle [see Fig. 2(a) and (c)]. Similar models have been used in previous studies of gait initiation [17], as well as more general types of movement [18]. The suitability of this type of model is supported by previous studies which have demonstrated 1) the predominant role played by the "ankle strategy" in providing postural stabilization [4], [19], [20] and 2) the relatively small magnitude of stance-leg hip rotation that occurs during compensatory step initiation [3]. Nonetheless, it should be noted that effects of swing-limb motion on the body dynamics were not included in the model, nor were potential contributions due to motion of the upper extremities.

To allow comparison with experimental data, it was assumed that the postural perturbation was generated via a-p support-surface (platform) acceleration $(a(t))$. The swingphase equations of motion are as follows [see Fig. 2(c) and the Nomenclature for definition of terms]

$$
\begin{aligned}
J \frac{d^{2} \Theta(t)}{d t}= & m g L \sin \Theta(t)-m L a(t) \cos \Theta(t)-T(t) \\
F_{y}(t)= & m a(t) \\
& +m L\left(\frac{d^{2} \Theta(t)}{d t^{2}} \cos \Theta(t)-\frac{d \Theta(t)^{2}}{d t} \sin \Theta(t)\right) \\
F_{z}(t)= & m g \\
& -m L\left(\frac{d^{2} \Theta(t)}{d t^{2}} \sin \Theta(t)+\frac{d \Theta(t)^{2}}{d t} \cos \Theta(t)\right)
\end{aligned}
$$

For each trial, the stance-leg ankle moment (or "torque"), $T(t)$, was set equal to the average value measured during the swing phase, as noted earlier. The a-p displacement and velocity of 


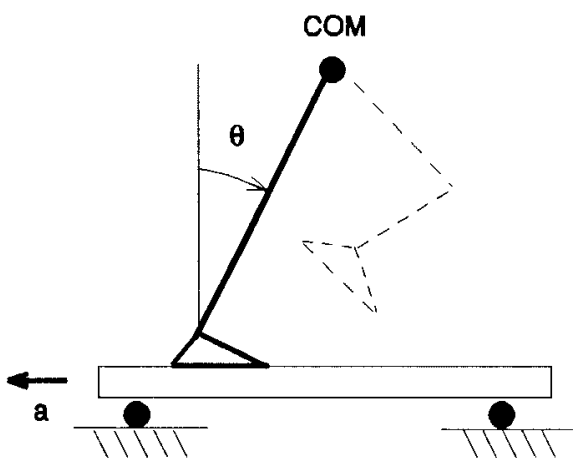

(a)

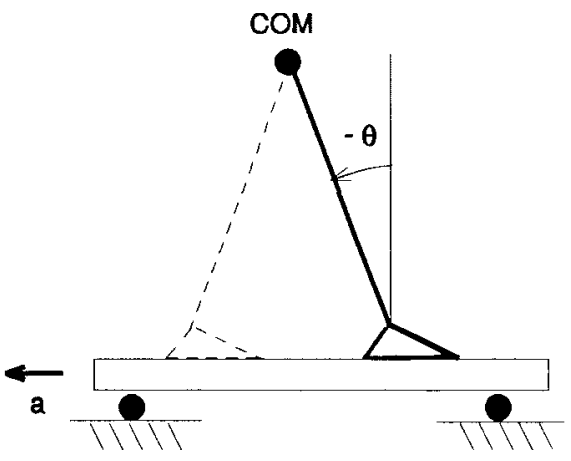

(b)

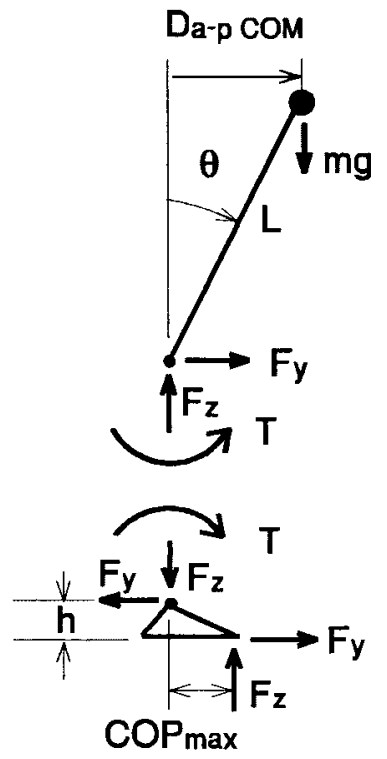

(c)

Fig. 2. The biomechanical model: (a) swing-phase dynamics, (b) postcontact dynamics, and (c) corresponding free-body diagram (diagram $\mathrm{C}$ applies to both models $\mathrm{A}$ and $\mathrm{B}$; variable definitions are listed at the start of the paper). Panels A and B depict a forward step, but the same model also applies for backward steps. Model A is used in extrapolating the trajectory of the center of mass (COM) past the recorded foot-contact (FC) time, in order to examine potential effects of delay in FC; COM position and velocity at time of FC are the "initial" conditions for these simulations. Model B is used in estimating the maximum COM velocity that could be decelerated, given the "initial" (time of FC) COM and swing-foot positions (to estimate the maximum decelerational capacity, it is assumed that all load is borne by the contacting foot, and that the COP is displaced to the anterior limit of this foot, i.e., $\mathrm{COP}_{\max }$ ). Note: effects due to the mass and inertia of the supporting foot were assumed to be negligible; effects of the motion of the nonsupporting leg on the body dynamics were also neglected.

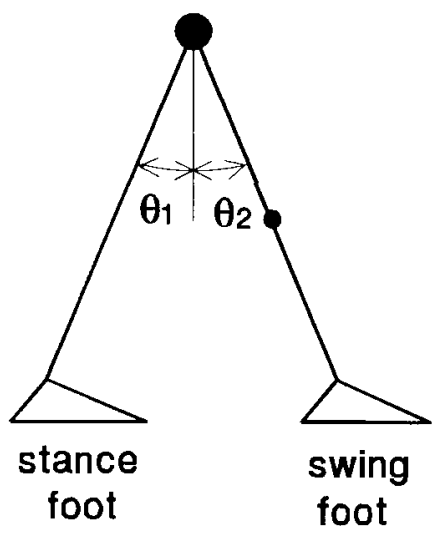

(a)

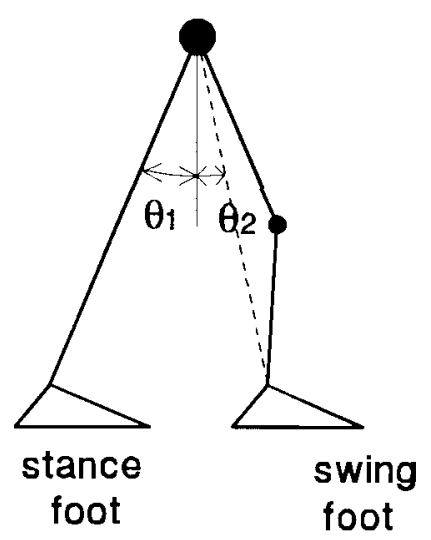

(b)

Fig. 3. Constraints on step length. Drawings depict a forward step for cases where the step length is (a) or is not (b) limited by geometrical requirements for foot-contact (if $\Theta_{2}>\Theta_{1}$, then foot-contact cannot occur). In addition to the geometrical constraints shown, the following range-of-motion limit was imposed: $\left|\Theta_{1}\right|<40^{\circ}$.

the COM, relative to the ankle axis of the stance foot, are

$$
\begin{aligned}
D_{\mathrm{a}-\mathrm{p} \mathrm{COM}}(t) & =L \sin \Theta(t) \\
V_{\mathrm{a}-\mathrm{p} \mathrm{COM}}(t) & =L \cos \Theta(t) \frac{d \Theta(t)}{d t} .
\end{aligned}
$$

For each potential FC time, the a-p BOS limit established by the swing foot (relative to the ankle axis of the stance foot) was defined as

$$
D_{\text {limit }}(t)=D_{o}+S_{\text {swing }}\left(t-t_{\mathrm{FO}}\right)
$$

where $D_{o}$ is the initial BOS limit (prior to FO), $S_{\text {swing }}$ is the mean a-p speed of the swing-foot movement (relative to the platform), $t$ is the $\mathrm{FC}$ time and $t_{\mathrm{FO}}$ is the $\mathrm{FO}$ time. To meet geometrical requirements for achieving foot-contact, the following step-length constraint was imposed:

$$
D_{\text {limit }}(t) \leq D_{o}+2 L \sin \Theta(t)
$$

(see Fig. 3). In addition, to ensure that the simulated step length did not exceed typical range-of-motion limits at the ankle or hip, the maximum angular displacement of the inverted pendulum, $\Theta(t)$, was limited to $\pm 40^{\circ}$. This limit was estimated from maximal step-length measurements performed on five male and four female subjects (ages 25-53). 


\section{B. Postcontact Simulation}

The results of the swing-phase simulation, as detailed above, were used to determine the DSM for each potential FC time $t$, i.e., the difference between $D_{\text {limit }}(t)$ and $D_{\text {a-p COM }}(t)$ [see (1)]. In order to determine the VSM, which was defined, in an analogous manner, as the difference between $V_{\text {limit }}(t)$ and $V_{\mathrm{a}-\mathrm{p} C O M}(t)$ [see (2)], it was necessary to simulate the postFC dynamics. The post-FC simulation was used to determine $V_{\text {limit }}(t)$, i.e., the maximum COM velocity that could have been decelerated at each potential FC time. The post-FC dynamics were approximated by a single-link inverted pendulum, with maximum stabilizing moment generated at the swingfoot ankle. To estimate the maximum ankle moment, it was assumed that the COP was displaced to the functional limit of the BOS established by this foot (the functional BOS length was defined to be equal to $75 \%$ of the anatomical foot length [21])

$$
T(t)=T_{\max }(t)=h F_{y}(t)+\mathrm{COP}_{\max } F_{z}(t) .
$$

The model is illustrated in Fig. 2(b). The free-body diagram shown in Fig. 2(c) and equations of motion (4)-(8) still apply, the only difference being that the sign of $\Theta$ is now inverted. The relative positioning of the BOS and COM at FC predicted by the swing-phase simulation were used to establish the initial value for $\Theta$ for each post-FC simulation. An iterative binarysearch approach was then used to determine the maximum initial (FC) velocity that could have been decelerated, given this initial $\Theta$. The criterion for finding this maximum requires that the COM motion is completely arrested at the instant that the COM reaches the BOS limit.

\section{MATERIALS AND MethodS}

Experimental data were needed to provide the initial conditions for the model simulations, as detailed in the previous section. In addition, comparisons between experimental measurements and model predictions were needed to test the validity of the model and to address the primary research questions posed in the Introduction. The experimental data were collected in two previous studies in which the compensatory stepping responses of healthy younger and older adults were compared [8], [22]. The experimental methods are outlined below. For more details, see the previous articles.

\section{A. Subjects}

Twenty volunteers participated in the studies: eight youngeradult subjects (22-28 years; four males and four females; weight $46-87 \mathrm{~kg}$, mean $=67 \mathrm{~kg}$; height $155-187 \mathrm{~cm}$, mean $=$ $170 \mathrm{~cm}$ ) and 12 older-adult subjects (65-81 years; seven males and five females; weight $43-99 \mathrm{~kg}$, mean $=67 \mathrm{~kg}$; height $152-182 \mathrm{~cm}$, mean $=167 \mathrm{~cm}$ ). Each subject provided written informed consent to comply with ethics approval granted by the institutional review board. Only subjects who had no prior exposure to balance experiments were recruited. None of the subjects reported significant neurologic, sensorimotor or musculoskeletal problems, persistent problems with dizziness, unsteadiness or falling, or use of medications that might affect balance. All older-adult subjects were active communitydwellers who did not use any mobility aids. Each subject, young and elderly, reported participating in some moderate physical activity (including walking) at least once per week.

\section{B. Protocol}

Forward and backward stepping movements were evoked by a-p translation of a moveable platform on which the subject stood [23]. The perturbation waveform comprised a 300-ms square-wave pulse of acceleration followed immediately by a $300-\mathrm{ms}$ deceleration pulse. The acceleration was $1.5 \mathrm{~m} / \mathrm{s}^{2}$ (velocity $0.45 \mathrm{~m} / \mathrm{s}$, displacement $0.135 \mathrm{~m}$ ) for forward translations (which evoked backward steps) and $2.0 \mathrm{~m} / \mathrm{s}^{2}$ (velocity 0.6 $\mathrm{m} / \mathrm{s}$, displacement $0.18 \mathrm{~m}$ ) for backward translations (which evoked forward steps). These waveform characteristics were selected, on the basis of prior experiments, to ensure that stepping responses were evoked. Subjects were provided with a demonstration of the platform motion prior to the first trial. Ten consecutive platform-translation perturbations were then presented, five forward and five backward (six subjects performed an additional backward trial). The direction of platform translation was randomized and there was a variable delay between successive trials ranging from 30-45 s. A standard initial foot position $\left(12^{\circ}\right.$ angle between the medial margins of the feet, $18-\mathrm{cm}$ spacing between the heels) was used in all trials [24]. Subjects were instructed to hold their arms at their sides, to look straight ahead at a visual target (1 $\mathrm{m}$ away) and to "try to keep from falling." They were given no specific instructions regarding foot motion.

\section{Measurements}

Ground reaction forces were sampled (at a rate of $200 \mathrm{~Hz}$ ) from two forceplates mounted side by side on the platform (for six subjects, a third forceplate, located behind the subject, was also used), and four high-resolution video cameras (shuttered at $1 / 500 \mathrm{~s}$ ) and recorders were used to record the motion of reflective markers placed on the limbs, trunk and head. The step location was determined (to within $1 \mathrm{~cm}$ ) by resolving the position of a marker on the foot relative to a grid marked on the platform, using an overhead camera view. The forceplate signals were used to determine the step timing, COM and COP displacement, and ankle moment. FO was defined to occur when the loading on the swing-foot forceplate dropped to less than $1 \%$ of body weight. FC was defined in a similar manner for steps where the foot landed on a forceplate $(88 \%$ of trials). For the remaining trials, the video recordings were used, in combination with the stance-leg forceplate data, to estimate the time of FC, as follows: video was first used to identify a time window ( \pm 2 frames, or $33 \mathrm{~ms}$ ) within which FC occurred, and the inflection point in the stance-leg vertical force that occurred within this time window was then used to pinpoint the timing. These latter FC estimates were accurate to within $\pm 18 \mathrm{~ms}$ (error SD), based on comparison with direct recordings of contact-onset for a subset of 36 trials where forceplate contact actually occurred.

A-p COM velocity was determined by integrating the a$\mathrm{p}$ ground reaction shear force, and COM displacement was 
determined by performing a second integration (the initial COM position was estimated by averaging the a-p COP during $500 \mathrm{~ms}$ of stationary standing immediately prior to the onset of the stimulus). Comparison with kinematic COM estimates (Peak Performance, Inc.; 15-segment model, using 31 body and limb markers), for a subset of 64 trials, showed reasonably good agreement for both displacement and velocity estimates. For COM displacement, the mean and standard deviation of the difference between the kinematic and forceplate estimates (at FC) were $0.008 \mathrm{~m}(4.7 \%)$ and $0.014 \mathrm{~m}(9.0 \%)$, respectively. For COM velocity, the corresponding values were $0.001 \mathrm{~m} / \mathrm{s}$ $(2.0 \%)$ and $0.039 \mathrm{~m} / \mathrm{s}(13.9 \%)$, respectively.

\section{Analysis}

For each trial, simulations, based on (4)-(11), were performed to determine the swing-phase durations corresponding to minimal requirements for stability and optimization of stability, for both DSM and VSM. In addition, the maximal DSM and VSM were determined. Experimentally determined values for the a-p COM displacement and velocity at actual time of FC, average a-p swing-foot velocity (a-p step distance/swing duration), and average swing-phase stance-leg ankle moment were used as inputs for the biomechanical simulations performed for that trial. Estimates of the inertial body parameters needed for the simulations were derived using measurements of the subject height and weight [25]. The simulations were performed by using Gear and Adams-Moultan multivalue methods [26] to integrate the equations of motion.

The observed swing durations were compared to model predictions of the timing required to meet both minimal and optimal requirements for stability. Similarly, the sizes of the observed stability margins were compared to the maximal DSM and VSM values predicted by the model. Paired $t$ tests were performed to determine whether any observed differences, between experimental and predicted values, were statistically significant $(\alpha=0.05)$. Separate analyzes were performed for trials where subjects recovered equilibrium by means of a single step $(n=71)$ and for trials where an additional step was used to further increase the a-p length of the BOS $(n=25)$. Multiple-step trials in which the second step did not provide such stabilization (e.g., lateral steps, steps in the opposite direction) were excluded from the analysis $(n=98)$. Also excluded were seven trials where subjects did not step and five trials where the stepping responses were predicted to be "unnecessary," i.e., the model indicated that the stance-leg ankle moment was sufficient to stabilize the COM over the initial BOS, without stepping (meaningful DSM and VSM values cannot be determined for such trials). In addition to the above analyzes, differences in the characteristics of the single- and multiple-step responses were analyzed by means of repeated-measures analysis of variance (ANOVA), and the Fisher Exact Test was used to examine the association between predictions of instability (i.e., VSM < 0) and multiple-step responses. Although age-related differences were not explicitly addressed in the present analyzes, it can be noted that the older subjects were much more likely to take multiple steps: $40 \%$ (17/42) of analyzed trials in the older group versus $15 \%(8 / 54)$

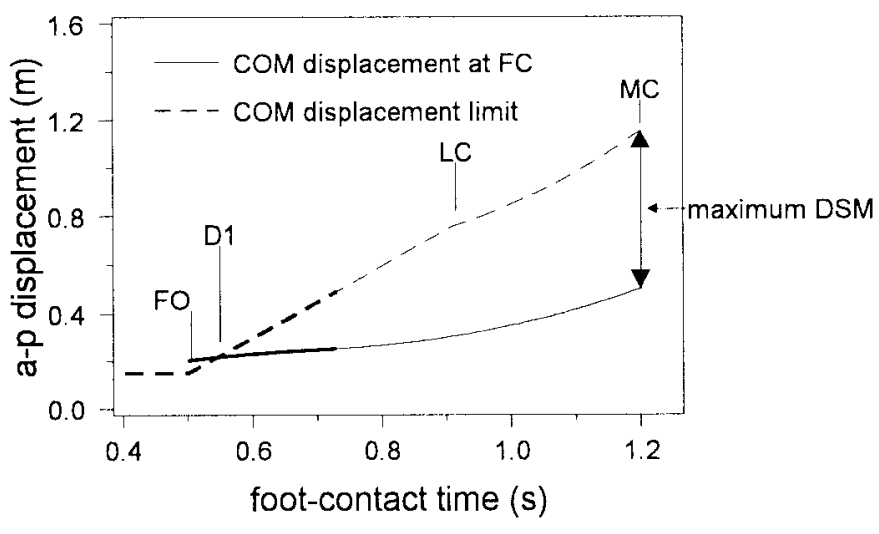

(a)

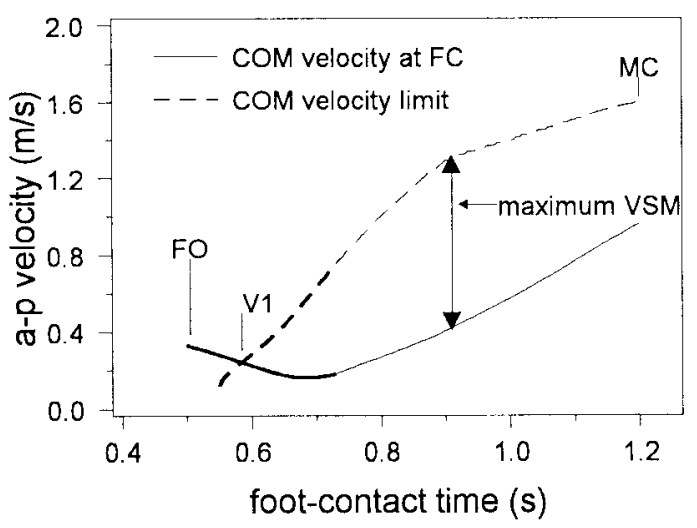

(b)

Fig. 4. Example model predictions, forward-step reaction (single trial): (a) displacement stability margin (DSM) and (b) velocity stability margin (VSM). Results are shown for all possible foot-contact (FC) times, ranging from foot-off (FO) to the time at which the maximum range-of-motion constraint (MC) is reached $\left(\Theta=40^{\circ} \mathrm{deg}\right)$. In panel $\mathrm{A}$, the DSM corresponding to any given $\mathrm{FC}$ time is equal to the distance between the a-p center-of-mass (COM) position (solid line) and the base-of-support (BOS) stability limit (broken line), i.e., the anterior end of the contacting-foot. LC indicates the point at which the step-length constraint begins to limit the size of the BOS; from FO to LC, the BOS size is limited only by the swing-foot velocity. D1 indicates the minimum FC time that would yield a stable DSM. In panel B, the VSM corresponding to any given FC time is equal to the difference between the COM velocity (solid line) and the maximum velocity that could have been decelerated (broken line), given the COM position and BOS limit occurring at that FC time. V1 indicates the minimum FC time that would yield a stable VSM. In both A and $\mathrm{B}$, the bold portion of each trajectory corresponds to the measured data; the thinner portion corresponds to the simulated data, extrapolated beyond the measured FC time $(0.73 \mathrm{~s})$. Displacements are defined relative to the moving platform $(0.0=$ stance-foot ankle axis, forward is positive $)$; time zero corresponds to onset of platform acceleration $\left(>0.1 \mathrm{~m} / \mathrm{s}^{2}\right)$. Note that the model predicts an optimal VSM (FC time $\simeq 0.90 \mathrm{~s}$ ); however, the DSM increases monotonically with FC time.

in the young $(p<0.005)$; further details regarding age-related differences are provided in previous articles [8], [22].

\section{RESULTS}

\section{A. Predicted Relationship Between Speed of Response and Step Stability}

Example simulation results, for a forward step, are shown in Fig. 4. For the data shown, the model predicts that the FC time 


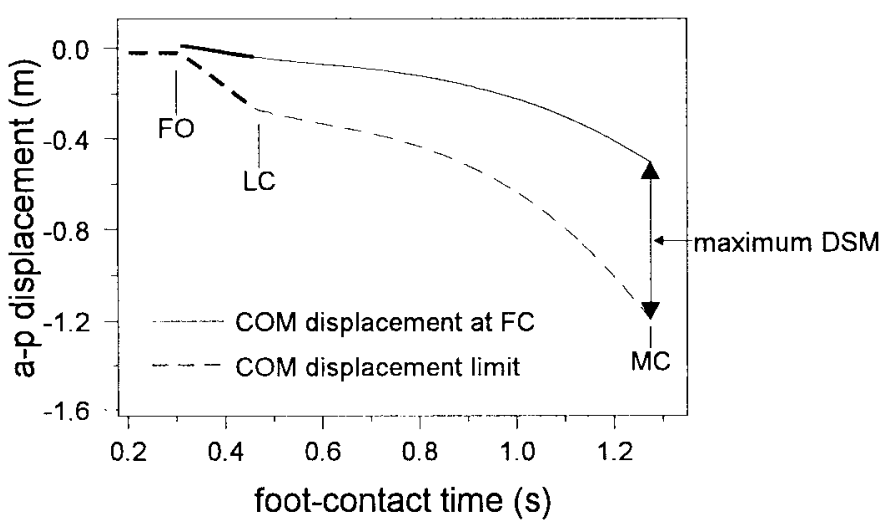

(a)

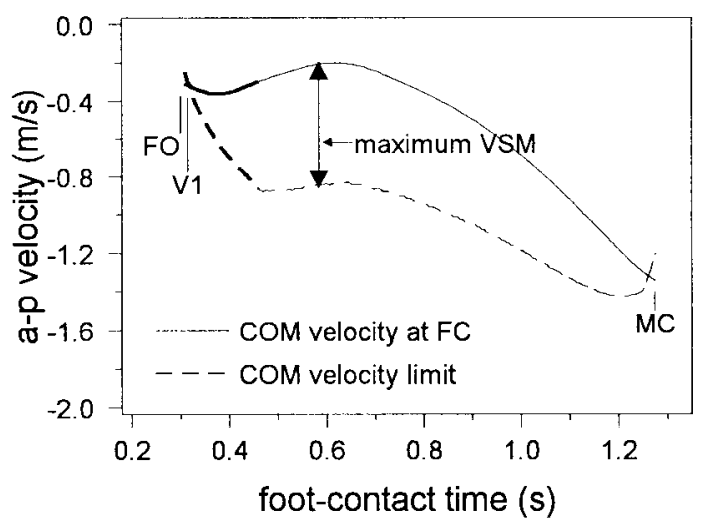

(b)

Fig. 5. Example model predictions, backward-step reaction. Layout and notation are the same as in Fig. 4, except that the BOS stability limit is now defined by the posterior, rather than anterior, end of the contacting-foot. In contrast to the trial shown in Fig. 4, there is no minimum requirement for FC time based on the DSM because foot-off occurs while the COM is still within the stability limits of the initial BOS.

(or, equivalently, the swing duration) must exceed a minimum value in order to meet requirements for a stable step. The DSM analysis establishes the minimum swing duration that will allow the new BOS established by the step to "capture" the COM [point D1 in Fig. 4(a)]; however, the VSM analysis indicates that the swing duration must be larger than this to ensure that the COM velocity can be decelerated [point V1 in Fig. 4(b)]. The VSM plot also predicts that there exists an optimal swing duration at which the VSM is maximized. The DSM, however, increases monotonically with increasing swing duration; hence, the maximum DSM occurs at the latest FC time that falls within the range-of-motion constraints. Example results for a backward step are shown in Fig. 5. The general form of the results is similar to that seen in the forward step, although it should be noted that the VSM tended to be smaller in the backward-step trials [compare also Fig. 6(b) and 6(d)]. Note also that the DSM plot for the trial shown does not exhibit a minimum requirement for swing duration, due to the fact that FO occurred while the COM was still located within the limits of the initial BOS (although the positioning of the COM might suggest that stepping was unnecessary, it should be noted that the COM velocity at time of FO did exceed the velocity stability limit).
In general, the DSM was not as useful as the VSM in establishing the limits of stability. The DSM analysis failed to predict a minimum nonzero swing-duration requirement for stability (because the COM was within the BOS limits at FO) in $82 \%(45 / 55)$ of backward-step trials and $41 \%(17 / 41)$ of forward-step trials. Conversely, as detailed below, the COM velocity exceeded the velocity stability limit at time of FO in $95 \%$ (91/96) of trials and the VSM analysis predicted a lower nonzero stable bound on the swing duration in all but four of these trials. For those trials where the DSM analysis did predict a nonzero lower bound, this limit was always superseded by the limit established by the VSM analysis. In addition, the DSM analysis failed to predict a true optimum for swing duration, whereas the VSM analysis almost invariably did predict an optimal swing duration that would maximize stability. Finally, the proposition that the CNS controls footcontact so as to center the COM over the BOS was not supported by the DSM data. On average, during single-step reactions, the DSM established by the swing foot was 0.02 $\mathrm{m}$ larger than the DSM defined with respect to the back (forward steps) or front (backward steps) of the stance foot $(p=0.002)$. For the initial step of multiple-step reactions, the DSM established by the swing foot was $0.12 \mathrm{~m}$ smaller than the DSM defined with respect to the opposite BOS boundary $(p<0.0001)$.

The VSM analysis indicated that the swing duration had to exceed a minimum nonzero value in order to achieve a stable step in 87 of 96 trials. In five trials, the step was predicted to be "globally stable," i.e., VSM $>0$ for all swing durations (within range-of-motion constraints). In the remaining four trials, the step was predicted to be "globally unstable" (VSM $<0$ for all possible swing durations; DSM $<0$ in one of these trials, also). A distinguishing feature of these latter responses, which all involved multiple steps, was the slow speed of the swing-foot movement $(0.79 \mathrm{~m} / \mathrm{s}$, on average, versus $1.76 \mathrm{~m} / \mathrm{s}$ in the other 92 trials), which resulted in a BOS that was inadequate to decelerate the COM. In all 92 trials that were not "globally unstable," there existed an optimal swing duration at which the VSM was maximized. The above results indicate that there typically does exist a tradeoff between the speed of response completion and the stability of the step. Increase in speed (reduction in swing duration) beyond a specific level is predicted to result in instability. Conversely, decrease in speed (increase in swing duration) is predicted to result in an increasing degree of stability, up to the point at which the optimal VSM is reached.

\section{B. Does Speed or Stability Take Precedence During Natural Stepping Behavior?}

Comparison of the experimentally observed responses to the stability limits predicted by the VSM model suggests that stability took precedence over speed of response in the majority of trials. For the 87 responses that had a nonzero lower stability limit, the model predicted that a stable step could be achieved, on average, by a swing duration as brief as 30 ms; however, all but two of these responses (85/87) exhibited 


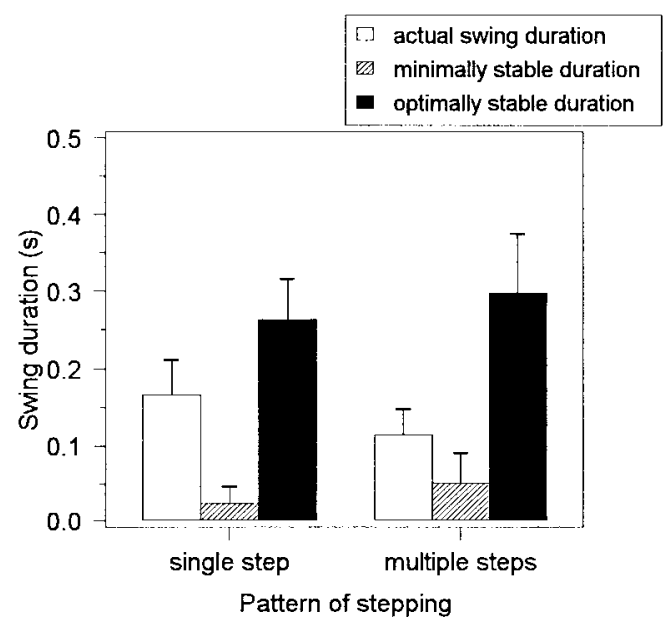

(a)

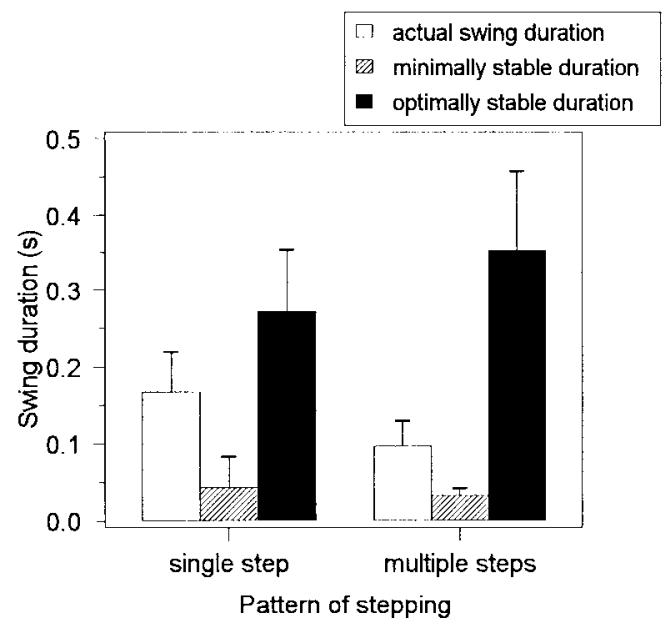

(c)

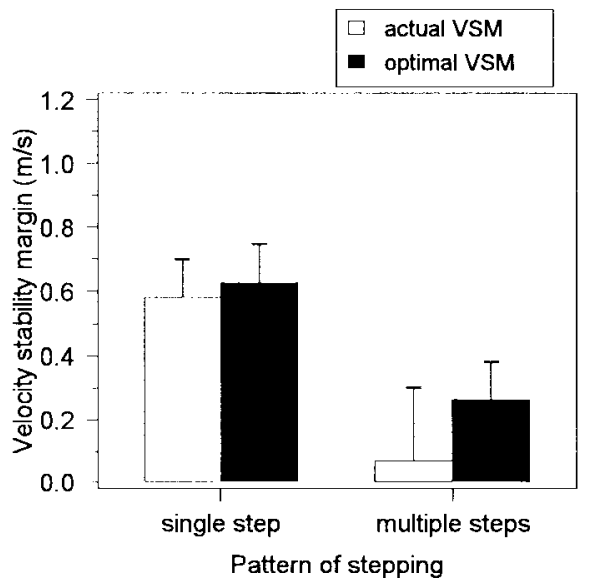

(b)

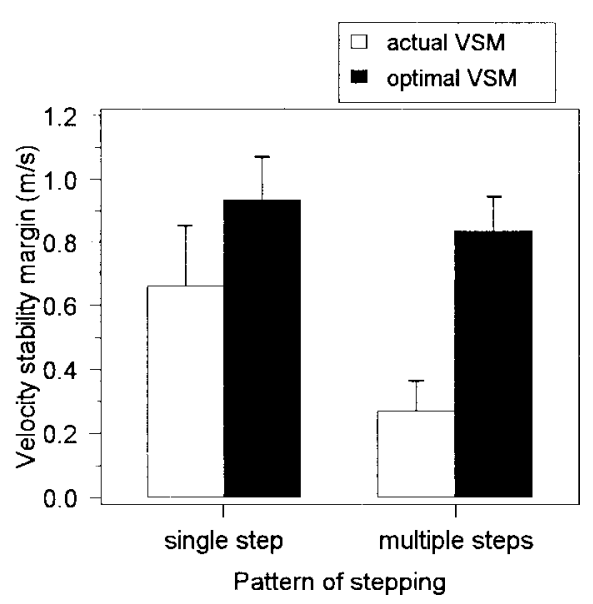

(d)

Fig. 6. Summary of velocity stability margin (VSM) results. Panel A compares, for backward steps, the experimentally measured swing duration (unfilled bars) with the critical values predicted by the model: 1) the minimally stable (VSM $=0$ ) swing duration (hatched bars) and 2) the optimally stable (VSM = maximum) swing duration (filled bars). Panel B compares the VSM corresponding to the measured swing duration (unfilled bars) with the maximum VSM predicted by the model (filled bars). Corresponding data, for forward-step reactions, are shown in panels C and D. In each panel, means (and standard deviations) are shown separately for the single-step reactions (37 backward steps, 34 forward steps) and for the initial step of the multiple-step reactions (18 backward steps, seven forward steps). Note the greater similarity between actual response and optimally stable values during single-step reactions; in contrast, the multiple-step reactions tended to involve more rapid steps and smaller levels of stability (VSM).

swing durations that exceeded the lower stability limit, and half of the observed swing durations (45/87) exceeded the lower limit by $120 \mathrm{~ms}$ or more. All of the latter steps were stable, as evidenced by the absence of further corrective action (i.e., multiple steps). For the 71 trials that involved stable (single-step) responses, the actual swing duration exceeded the minimally stable value predicted by the VSM model by $135 \mathrm{~ms}(s d=48)$, on average $[p<0.0001$; Fig. $6(\mathrm{a})$ and (c) $]$. Moreover, the size of the stability margin that was achieved was substantial. Although the observed single-step responses did not quite achieve the optimal levels of stability predicted by the model [Fig. 6(b) and (d)], the actual VSM was, on average, equal to $80 \%$ of the optimal value. In order to achieve the optimum VSM, the actual swing duration would have had to increase by $60 \%$ (100 ms), on average.

Although the swing duration in the single-step responses tended to exceed the minimally stable lower limit by a substantial margin, a tradeoff of step stability in favor of greater speed of response was evident in the 21 trials where multiple steps were used to increase the BOS. On average, the actual swing duration for the multiple-step responses exceeded the minimally stable value predicted by the VSM model by only $55 \mathrm{~ms}$, in contrast to the margin of $135 \mathrm{~ms}$ seen in the single-step responses; thus, the actual swing-duration was much closer to the minimum value required for stability [Fig. 6(a) and (c)]. Moreover, the VSM associated with the actual FC time was reduced by a factor of $81 \%$, on average, in comparing multiple- versus single-step responses $(0.12$ versus $0.62 \mathrm{~m} / \mathrm{s}$ ). In relative terms, the VSM occurring at the actual FC time was only $44 \%$ of the optimal VSM, in comparison to the ratio of $80 \%$ seen in the single-step responses. This relative change was less pronounced than the absolute change because the optimal VSM itself was also reduced during multiple-step reactions [particularly for backward steps; see Fig. 6(b)]. 


\section{Does Decrease in Stability Margin Predict the Need to Take Further Steps?}

There appeared to be a number of differences in the spatial-temporal characteristics, in comparing the initial step of multiple-step reactions to the steps occurring during the single-step reactions. In particular, the initial steps during multiple-step reactions tended, on average, to be shorter in length (7\% versus $18 \%$ of body height) and duration (109 versus $167 \mathrm{~ms})$ and were completed more rapidly (444 versus $579 \mathrm{~ms}$ ); a-p COM velocity was also higher (24\% versus $14 \%$ of body height/s at FC). None of these apparent trends, however, was statistically significant $(p$ 's $>0.10)$. In contrast, the difference in relative VSM magnitude noted above $(80 \%$ in single-step trials versus $44 \%$ in multiplestep trials) was significant $(p=0.013)$. In attempting to use the step characteristics and stability margin measures to predict single- and multiple-step reactions via stepwise logistic regression, the best predictor was found to be the DSM at actual time of FC (Wald chi-square, $p=0.003$; concordancy of predictions $=99.7 \%$ ). VSM at time of actual $\mathrm{FC}$ was also a very good predictor (Wald chi-square, $p=0.002$; concordancy of predictions $=98.1 \%$ ). In addition, there was a statistically significant association between unstable (negative) VSM and multiple-step reactions: multiple steps occurred in $83 \%$ of trials $(5 / 6)$ in which the VSM was negative at actual FC (initial step), but in only $21 \%(20 / 90)$ of reactions that had positive VSM values $(p<0.005)$.

\section{Accuracy of the Simulations}

In order to test the accuracy of the inverted-pendulum simulations, the model was used to predict the COM displacement and velocity at time of $\mathrm{FC}$, based on simulations starting at time of FO. These predictions were then compared to the measured values. Over the 96 experimental trials that were analyzed, the mean and standard deviation of the error between predictions and measurements of COM displacement were 0.004 and $0.006 \mathrm{~m}$, respectively. For COM velocity, the corresponding errors were 0.055 and $0.067 \mathrm{~m} / \mathrm{s}$, respectively. These errors are reasonably small, in comparison to the mean absolute COM displacement $(0.14 \mathrm{~m})$ and velocity $(0.28 \mathrm{~m} / \mathrm{s})$.

\section{DISCUSSION}

The results of the modeling performed in this study clearly indicate that there is a tradeoff between the speed of compensatory step execution and the stability of the resulting step. The model predicted that, for a given set of step characteristics (foot-off time, swing velocity, swing-phase COM trajectory), overly rapid completion of the step (e.g., swing duration $<30 \mathrm{~ms}$ ) would result in a BOS that is inadequate to decelerate the horizontal motion of the COM. Conversely, by extending the swing duration to approximately $270 \mathrm{~ms}$, it is possible to achieve a maximal level of stability. Typically, during single-step reactions, behavior appeared to be biased toward achieving stability more so than speed. By selecting a swing duration that was on average $135 \mathrm{~ms}$ longer than the minimally stable value, the stability of the step was increased dramatically, to about $80 \%$ of the optimal level predicted by the model. Optimal stability would require a further delay of about $100 \mathrm{~ms}$ in completing the step, whereas the additional benefit achieved, in terms of the increase in VSM, would only be $20 \%$.

It may be beneficial to avoid unnecessary delays in step completion for a number of reasons. A shorter swing duration may, for example, reduce the tendency of the COM to fall laterally during step execution [27]. In addition, as noted earlier, faster responses will facilitate successful execution of additional stepping reactions, should the initial step prove to be inadequate to recover equilibrium. The execution of additional steps, which was much more prevalent in the older adults, could be a result of errors or inadequacies in the planning or execution of the initial step (e.g., due to age-related changes in the neural, sensory or musculoskeletal systems); however, this could also be the consequence of a CNS control strategy to tradeoff relative stability in favor of greater speed of response. In fact, some have suggested that older adults may preferentially adopt such a strategy [7]. In either case, the reduction in VSM in the initial step of the multiple-step reactions is consistent with a biomechanical need to execute additional steps, and appears to support the validity of the modeling approach. That the VSM did not actually reach a negative (unstable) level in many of these steps is likely due to the fact the VSM analysis assumes an idealized, maximal capacity for COM deceleration (instantaneous transfer of all weight to the swing leg and instantaneous generation of maximal ankle moment, at time of FC) and thereby overestimates the true biomechanical capabilities.

The present results indicate that it is the capacity to decelerate the momentum of the falling body (reflected in the VSM), rather than the capacity to "capture" the COM within the BOS boundaries (reflected in the DSM), that ultimately defines the stability of the step. Nonetheless, the DSM, which quantifies the relative displacement of the COM and BOS, is important because this defines the maximum decelerational impulse that can be generated, a key factor that influences the VSM. This connection may explain why the DSM was found to be as good a predictor of multiple-step reactions as the VSM. The VSM is, to our knowledge, a novel measure for quantifying the dynamic stability associated with the capacity to decelerate the COM. It should be noted, however, that our approach is completely consistent with recent work by Pai and Patton [18], who have used a similar biomechanical model to identify feasible combinations of COM position and velocity that will allow balance to be maintained during movement termination.

It remains to be determined how the control of the stepping reaction is accomplished by the CNS. Redfern and Schumann [13] have proposed that the control of stepping during gait involves a symmetrical positioning of the swing foot and stance foot with respect to the pelvis at time of foot-contact, and have suggested that the CNS could implement such a control scheme largely on the basis of proprioceptive feedback from the hip and knee joints and associated musculature. The present results suggest that such a model may not apply to compensatory stepping reactions, indicating instead that the positioning of the COM with respect to the BOS established by 
the step is typically biased rather than centered: a larger DSM in the direction of the fall, as observed, apparently serves to increase the capacity to decelerate the falling motion of the body (single-step reactions), whereas bias in the opposite direction tends to occur when speed of response takes precedence over stability (multiple-step reactions). Hip and knee proprioception could still be important, although recent results indicate that feedback from cutaneous mechanoreceptors, on the plantar foot surface, may also play a critical role in regulating these reactions [28]-[32].

Although control of stability during stepping requires regulation of both COM and BOS, there has been a tendency, in many studies, to address the control of the COM without giving explicit consideration to the factors that govern the control of the foot placement, e.g., [33]-[35]. It has been noted, by some, that foot-placement control actually appears to be the most important factor affecting stability [11]-[13], [34]. Those models that have addressed the regulation of the foot trajectory have focussed on volitional or gait-related tasks [10]-[16], but none have considered control of compensatory stepping reactions. Our findings, as already noted, indicate that the need to decelerate the momentum of the falling COM during compensatory reaction leads to significant alterations in the control of the foot placement.

The model of foot-placement control developed in the current study is based on the premise that control of step stability and speed of response is likely to take precedence over other control objectives when stepping to recover balance. In contrast, previous models pertaining to the control of gait or obstacle avoidance have tended instead to focus on energy expenditure [14], [15], [36], [37]. Avoidance of fatigue is of course important during repetitive movements such as gait, but is less likely to be a predominant concern in responding to transient events, particularly when these events pose a substantial threat to stability and safety. The general principle that safety takes priority is supported by findings that observed clearances, in stepping over obstacles, are significantly larger than the trajectories predicted by energy-minimization models [15].

Other factors that could potentially influence the regulation of compensatory stepping reactions pertain to the contact forces occurring during landing. Excessive demand for shear force at the contact foot could, for example, increase the risk of slipping. Impact absorption may also be important, not only in avoiding musculoskeletal injury, but also in controlling the stability of the landing. The latter is supported, indirectly, by apparent associations between high rates of contact loading (during the initial step) and the need to take additional steps to recover equilibrium [22], [31]. Recent modeling work has demonstrated that step length can be controlled so as to minimize the impact force [38]; however, more research is need to determine whether control of contact loading can provide further insight, beyond the results presented here, to explain the selection of step parameters that occurs during natural compensatory stepping behavior.

To simplify the model, the present study was restricted to analysis of the effects of a single step parameter: swing duration. By studying the effects of varying swing duration while holding all other parameters constant, we were able to investigate whether the selected swing duration was consistent with efforts to maximize either speed or stability. Of course, control of the other step parameters, such as timing of step initiation and selection of the swing velocity, is also important and future modeling efforts, will be directed at investigating these parameters. In addition, future models will include consideration of lateral, as well as antero-posterior stability.

A potential limitation of the present model pertains to the use of a single-link inverted pendulum model to approximate the supporting-leg dynamics. Comparisons between measured and predicted COM trajectories did, however, suggest that the simplifications inherent to the model did not lead to large errors. Another limitation, as noted above, pertains to the fact that the VSM estimates were based on several idealized assumptions regarding weight transfer and moment generation. The veracity of the model could likely be improved in future work by replacing the simplifying assumptions with more realistic estimates, or measurements, of actual behavior. With regard to the experimental data, it should be noted that the multiple-step reactions that were considered in the analysis were restricted to those that showed the most definitive evidence of a-p instability during the initial step; i.e., the second step actually increased the a-p length of the BOS in the direction of the "fall." Further work is needed to understand the control of multiple-step reactions that involve other patterns of stepping [8].

In summary, the biomechanical model presented in this paper demonstrates that there is a tradeoff between the speed of compensatory step execution and the stability of the resulting step: there typically exists a minimum swing duration that maximizes speed of response while meeting minimum requirements for static and dynamic stability (DSM and VSM $\geq 0$ ), and there also exists a slower speed of response (longer swing duration) at which dynamic stability (VSM) is maximized. Experimental data indicate that stability of the step typically takes precedence over speed of response completion during natural behavior, except in reactions where additional steps are taken to recover equilibrium. The reduction in VSM in these latter reactions is consistent with a need to execute additional steps, and appears to support the validity of the modeling approach. The modeling approach developed in this study may help to provide new insight into the biomechanical factors that govern the neural control of compensatory stepping reactions.

\section{ACKNOWLEDGMENT}

The authors would like to thank G. Fernie for suggestions and comments, G. Griggs for technical support, and M. A. Edmondstone, N. Jiang, D. Lawless, and S. Perry for assistance in data collection, data processing, and/or manuscript preparation. They also acknowledge the contribution of T. Sinha to the preliminary stages of the model development.

\section{REFERENCES}

[1] W. E. McIlroy and B. E. Maki, "Task constraints on foot movement and the incidence of compensatory stepping following perturbation of upright stance," Brain Res., vol. 616, pp. 30-38, 1993. 
[2] M. W. Rogers, T. C. Hain, T. A. Hanke, and I. Janssen, "Stimulus parameters and inertial load: effects on the incidence of protective stepping responses in healthy human subjects," Arch. Phys. Med. Rehabil., vol. 77, pp. 363-368, 1996.

[3] B. E. Maki and W. E. McIlroy, "The role of limb movements in maintaining upright stance: The "change-in-support" strategy," Phys. Ther., vol. 77, pp. 488-507, 1997.

[4] L. M. Nashner and P. J. Cordo, "Relation of automatic postural responses and reaction-time voluntary movements of human leg muscles," Exp. Brain Res., vol. 43, pp. 395-405, 1981.

[5] L. I. Wolfson, R. Whipple, P. Amerman, and A. Kleinberg, "Stressing the postural response: A quantitative method for testing balance," $J$. Amer. Geriatr. Soc., vol. 34, pp. 845-850, 1986.

[6] J. M. Chandler, P. W. Duncan, and S. A. Studenski, "Balance performance on the postural stress test: Comparison of young adults, healthy elderly, and fallers," Phys. Ther., vol. 70, pp. 410-415, 1990.

[7] C. W. Luchies, N. B. Alexander, A. B. Schultz, and J. Ashton-Miller, "Stepping responses of young and old adults to postural disturbances: Kinematics," J. Amer. Geriatr. Soc., vol. 42, pp. 506-512, 1994.

[8] W. E. McIlroy and B. E. Maki, "Age-related changes in compensatory stepping in response to unpredictable perturbations," J. Gerontol., vol. 51A, pp. M289-M296, 1996.

[9] D. G. Thelen, L. A. Wojcik, A. B. Schultz, J. A. Ashton-Miller, and N. B. Alexander, "Age differences in using a rapid step to regain balance during a forward fall," J. Gerontol., vol. 52A, pp. M8-M13, 1997.

[10] H. Flashner, A. Beuter, and A. Arabyan, "Modeling of control and learning in a stepping motion," Biol. Cybern., vol. 55, pp. 387-396, 1987.

[11] F. Gubina, H. Hemami, and R. B. McGhee, "On the dynamic stability of biped locomotion," IEEE Trans. Biomed. Eng., vol. BME-21, pp. 102-108, 1974.

[12] M. A. Townsend, "Biped gait stabilization via foot placement," $J$. Biomech., vol. 18, pp. 21-38, 1985.

[13] M. S. Redfern and T. Schumann, "A model of foot placement during gait," J. Biomech., vol. 27, pp. 1339-1346, 1994.

[14] L. S. Chou, S. M. Song, and L. F. Draganich, "Predicting the kinematics and kinetics of gait based on the optimum trajectory of the swing limb," J. Biomech., vol. 28, pp. 377-385, 1995.

[15] L. S. Chou, L. F. Draganich, and S. M. Song, "Minimum energy trajectories of the swing ankle when stepping over obstacles of different heights," J. Biomech., vol. 30, pp. 115-120, 1997.

[16] M. Armand, J. P. Huissoon, and A. E. Patla, "Stepping over obstacles during locomotion: Insights from multiobjective optimization on set of input parameters," IEEE Trans. Rehab. Eng., vol. 6, pp. 43-52, 1998.

[17] Y. Jian, D. A. Winter, M. G. Ishac, and L. Gilchrist, "Trajectory of the body COG and COP during initiation and termination of gait," Gait Posture, vol. 1, pp. 9-22, 1993.

[18] Y. C. Pai and J. Patton, "Center of mass velocity-position predictions for balance control," J. Biomech., vol. 30, pp. 347-354, 1997.

[19] H. C. Diener, F. B. Horak, and L. M. Nashner, "Influence of stimulus parameters on human postural responses," J. Neurophysiol., vol. 59, pp. $1888-1905,1988$

[20] W. E. Mcllroy and B. E. Maki, "Changes in early 'automatic' postural responses associated with the prior-planning and execution of a compensatory step," Brain Res., vol. 631, pp. 203-211, 1993.

[21] C. L. Riach and J. L. Starkes, "Stability limits of quiet standing postural control in children and adults," Gait Posture, vol. 1, pp. 105-111, 1993.

[22] B. E. Maki and W. E. McIlroy, " Control of compensatory stepping reactions: Age-related impairment and the potential for remedial intervention," Physiotherapy Theory and Practice, to be published.

[23] B. E. Maki, W. E. McIlroy, and S. D. Perry, "Influence of lateral destabilization on compensatory stepping responses," J. Biomech., vol. 29, pp. 343-353, 1996

[24] W. E. McIlroy and B. E. Maki, "Preferred placement of the feet during quiet stance: Development of a standardized foot placement for balance testing," Clin. Biomech., vol. 12, pp. 66-70, 1997.

[25] D. A. Winter, Biomechanics of Human Movement. New York: WileyInterscience, 1979.

[26] D. Kahaner, C. Moler, and S. Nash, Numerical Methods and Software. Englewood Cliffs, NJ: Prentice-Hall, 1989.

[27] W. E. McIlroy and B. E. Maki, "The control of lateral stability during rapid compensatory stepping reactions: Does anticipatory control play a role?" Gait Posture, to be published.

[28] M. C. Do, B. Bussel, and Y. Breniere, "Influence of plantar cutaneous afferents on early compensatory reactions to forward fall," Exp. Brain Res., vol. 79, pp. 319-324, 1990.
[29] M. C. Do and A. Roby-Brami, "The influence of a reduced plantar support surface area on the compensatory reactions to a forward fall," Exp. Brain Res., vol. 84, pp. 439-443, 1991.

[30] S. D. Perry and B. E. Maki, "The role of cutaneous mechanoreceptors in the control of compensatory stepping," presented at Canadian Society for Biomechanics, Burnaby, Canada, 1996.

[31] B. E. Maki, S. D. Perry, R. G. Norrie, and W. E. McIlroy, "Effect of facilitation of sensation from plantar foot-surface boundaries on postural stabilization in young and older adults," J. Gerontol., to be published.

[32] S. D. Perry, W. E. McIlroy, and B. E. Maki, "The role of cutaneous mechanoreceptors in the control of compensatory stepping reactions evoked by unpredictable, multi-directional perturbation," to be published

[33] J. F. Yang, D. A. Winter, and R. P. Wells, "Postural dynamics of walking in humans," Biol. Cybern., vol. 62, pp. 321-330, 1990.

[34] C. D. MacKinnon and D. A. Winter, "Control of whole body balance in the frontal plane during human walking," J. Biomech., vol. 26, pp. 633-644, 1993.

[35] K. Iqbal, H. Hemami, and S. Simon, "Stability and control of a frontal four-link biped system," IEEE Trans. Biomed. Eng., vol. 40, pp. 1007-1017, 1993

[36] D. A. Winter and D. G. Robertson, "Joint torque and energy patterns in normal gait," Biol. Cybern., vol. 29, pp. 137-142, 1978.

[37] K. G. Holt, J. Hamill, and R. O. Andres, "Predicting the minimal energy costs of human walking," Med. Sci. Sports. Exerc., vol. 23, pp. 491-498, 1991.

[38] E. T. Hsiao, V. Frenk, and S. N. Robinovitch, "Stepping mechanics utilized in balance recovery: Swing and contact phase modeling," presented at 20th Annual Meeting of the American Society of Biomechanics, 1996.

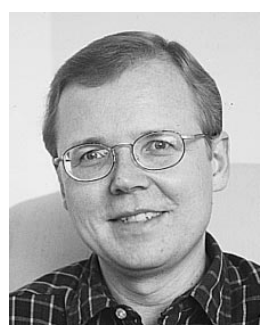

Brian E. Maki (M'93) received the B.A.Sc. degree in mechanical engineering from the University of British Columbia, Canada, in 1979, the Masters degree in mechanical engineering from the Massachusetts Institute of Technology, Cambridge, in 1982, and the Doctoral degree in bioengineering from the University of Strathclyde, Glasgow, U.K. in 1987.

In 1979, he worked in Biomedical Engineering at Vancouver General Hospital. He has worked at the Centre for Studies in Aging, Sunnybrook Health Science Centre, Toronto, Ont., Canada, since 1982. He is currently an Associate Professor at the University of Toronto, Toronto, Ont., Canada, and a Senior Scientist at Sunnybrook and Women's College Health Sciences Centre. His primary research interests involve the biomechanics and control of human posture and movement, and his research has focused on the problem of falling in older adults, in particular, understanding the contribution of age-related deterioration in postural control to an increased risk of falling, development of improved methods for assessing postural balance and predicting falling risk, and development of novel interventions to prevent falls and related injuries.

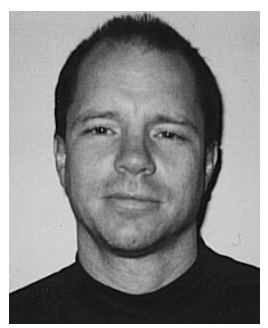

William E. McIlroy received the Ph.D. degree in neurophysiology from the University of Guelph, Guelph, Ont., Canada, in 1991.

$\mathrm{He}$ was a postdoctoral fellow at the Centre for Studies in Aging, Sunnybrook Health Science Centre, Toronto, Toronto, Ont., Canada, from 1992 to 1996. In 1996, he joined the Department of Physical Therapy and Graduate Department of Rehabilitation Science, University of Toronto, Toronto, Ont., Canada, as an Assistant Professor. He also continues as a Research Scientist at Sunnybrook and Women's College Health Sciences Centre. His research focuses on the central nervous system (CNS) control of human posture and movement. His specific interests include developing an understanding of the contribution of aging and neurologic disease to impaired postural and movement control. An important objective of his work is to improve basic understanding of CNS control, so as to permit the development of more effective therapeutic techniques and the development of novel assistive aids to maintain/improve sensorimotor function with advancing age and following neurologic injury. 